\title{
La negociación de un compromiso: la mita de las minas de plata de San Agustín de Huantajaya, Tarapacá, Perú (1756-1766)
}

La négociation d'un compromis : la mita des mines d'argent de San Agustín de Huantajaya, Tarapacá, Pérou (1756-1766)

Negotiating a compromise: the mita at the silver mines of San Agustin de Huantajaya, Tarapaca, Peru (1756-1766)

\section{Anil Mukerjee}

\section{(2) OpenEdition} Journals

Edición electrónica

URL: http://journals.openedition.org/bifea/3435

DOI: 10.4000/bifea.3435

ISSN: 2076-5827

\section{Editor}

Institut Français d'Études Andines

\section{Edición impresa}

Fecha de publicación: 1 abril 2008

Paginación: 217-225

ISSN: 0303-7495

Referencia electrónica

Anil Mukerjee, «La negociación de un compromiso: la mita de las minas de plata de San Agustín de Huantajaya, Tarapacá, Perú (1756-1766) », Bulletin de l'Institut français d'études andines [En línea], 37 (1) | 2008, Publicado el 01 octubre 2008, consultado el 26 noviembre 2020. URL : http:// journals.openedition.org/bifea/3435; DOI : https://doi.org/10.4000/bifea.3435

Les contenus du Bulletin de l'Institut français d'études andines sont mis à disposition selon les termes de la licence Creative Commons Attribution - Pas d'Utilisation Commerciale - Pas de Modification 4.0 International. 


\title{
La negociación de un compromiso: la mita de las minas de plata de San Agustín de Huantajaya, Tarapacá, Perú (1756-1766)
}

\author{
Anil Mukerjee*
}

\section{Resumen}

Con excepción de las minas de Potosí y Huancavelica, la implementación del sistema de mita en las minas nunca tuvo mucho éxito a lo largo del periodo colonial. En 1758, la Corona concedió una mita al propietario de la mina de plata de San Agustín de Huantajaya (Tarapacá), la única zona minera en el Virreinato que no se encontraba en los Andes. La posibilidad de consultar el libro de registros del propietario de la mina, que en ocasiones recogía el intercambio diario de cartas entre el propietario de la mina y los curacas, nos proporciona una descripción mucho más detallada que lo normal sobre las negociaciones que tenían lugar en las minas donde la mita se implementaba. En lugar de observar un incremento en las políticas contenciosas que caracterizaban este periodo en Perú, lo que encontramos es una persistencia de las normas que gobernaban las relaciones entre los curacas y la sociedad colonial. Sin embargo, gracias en su mayor parte a los esfuerzos de los curacas de Tarapacá, la mita de esta mina nunca llegó a su fin y fue reasignada a una tarea que requería un menor esfuerzo que el extenuante trabajo en las minas.

Palabras clave: mita, minas de plata, curacas, San Agustín de Huantajaya, Tarapacá, Perú

\section{La négociation d'un compromis : la mita des mines d'argent de San Agustín de Huantajaya, Tarapacá, Pérou (1756-1766)}

\section{Résumé}

À l'exception des mines de Potosí et de Huancavelica, I'application du système de la mita dans les mines n'a pas connu un grand succès tout au long de la période coloniale. En 1758, la Couronne

* University of California, Santa Barbara (California). E-mail: anil@umail.ucsb.edu

Este texto está dedicado a Cecilia Méndez Gastelumendi, a quien agradezco por su orientación y atinados comentarios. Una versión preliminar de este texto fue leída en julio del 2006, en el 52을 Congreso Internacional de Americanistas en Sevilla (España). 
accorda un droit de mita au propriétaire de la mine d'argent de San Agustín de Huantajaya, Tarapacá, la seule zone minière de la Vice-Royauté qui n'était pas située dans les Andes. La possibilité de consulter le livre des registres du propriétaire de la mine, qui consignait l'échange parfois quotidien des lettres entre le propriétaire de la mine et les curacas, nous apporte une description beaucoup plus détaillée que d'habitude sur les négociations qui avaient lieu dans les mines où la mita était appliquée. Au lieu d'observer l'augmentation des contentieux politiques qui caractérisent cette période au Pérou, nous constatons une persistance des normes qui gouvernaient les relations entre les curacas et la société coloniale. De fait, grâce surtout aux efforts des curacas de Tarapacá, la mita de cette mine ne fut jamais appliquée et fut réattribuée à une tâche qui demandait un moindre effort que l'exténuant travail dans les mines.

Mots clés : mita, mines d'argent, curacas, San Agustín de Huantajaya, Tarapacá, Pérou

\title{
Negotiating a compromise: the mita at the silver mines of San Agustin de Huantajaya, Tarapaca, Peru (1756-1766)
}

\begin{abstract}
The implementation of the mining mita other than in Potosi and Huancavelica was never very successful during the colonial period. In 1758, the Spanish Crown awarded a mining mita to the owner of a silver mine in San Augustin de Huantajaya, Tarapacá, the only mining area in the viceroyalty that was not located in the Andes. The survival of a mine owner's registry book that recorded the sometimes daily exchange of letters between the mine owner and the kurakas permits a much more detailed view than is commonly available of the negotiations that took place when such mitas were implemented. Rather than seeing a rise in contentious politics that characterizes this period in Peru, we have a persistence of norms that governed kuraka relations with colonial society. Nevertheless, largely through the efforts of the kurakas of Tarapacá, this mining mita was never implemented and less strenuous tasks than the dreaded work in the mines were reassigned to the mita.
\end{abstract}

Key words: mita, silver mines, curacas, San Agustín de Huantajaya, Tarapacá, Perú

La mayor parte de los estudios dedicados a la historia del siglo XVIII en el Perú se ha centrado en intentar explicar el auge de los conflictos en el altiplano minero del Virreinato en los momentos previos a la rebelión de Túpac Amaru de 1781. La creciente demanda de trabajo indígena y recursos de la zona en un contexto de declive económico fueron factores importantes que provocaron la rebelión en estas zonas. Como resultado de la concentración casi exclusiva de dichos estudios en la escalada de dichas tensiones y la consiguiente directa confrontación entre la sociedad indiana y el Estado colonial, otras áreas del Perú que permanecieron imperturbadas en esos momentos han sido desatendidas por los historiadores. La provincia de Tarapacá, situada en una región desértica en la costa del Pacífico a unos mil kilómetros al sur de Lima, no experimentó ningún tipo de revuelta social durante este periodo a pesar de que el trabajo de los indígenas de esta región fue solicitado en numerosas ocasiones. El presente trabajo analiza los mecanismos mediante los cuales los kurakas de Tarapacá fueron capaces de evitar la implementación de una de dichas demandas: la mita en la mina; la que habría de someter a los indígenas al trabajo en una mina de plata en el área, una mita por la que nunca antes habían sido requeridos. 
La habilidad del kuraka para proteger a los indígenas de las nuevas demandas del Estado colonial sin alienar a las autoridades locales fue clave para la continuación de la estabilidad en la región. A la luz de la delicada situación política — siempre en paulatino estado de deterioro en otras partes del Virreinato-, la habilidad de estos kurakas resulta, si cabe, de mayor mérito.

Las minas de plata de San Agustín de Huantajaya en la provincia de Tarapacá eran las únicas minas del virreinato del Perú que no se encontraban situadas en la sierra. Huantajaya se encuentra en el desierto de Atacama, a 1000 m.s.n.m. y a 30 km (aprox.) hacia el interior de la actual ciudad portuaria de Iquique en Chile. Sin ser comparable a la riqueza de las minas del Potosí, Huantajaya fue una importante fuente de plata durante el periodo colonial, llegando a producir el $10 \%$ de la plata del Virreinato. Los asentamientos indígenas más cercanos se encontraban a más de $100 \mathrm{~km}$ hacia el este en las estribaciones andinas. Desde esos pequeños asentamientos localizados en los repartimientos de Tarapacá y Sibaya, se reclutaban a los indígenas para la mita de la mina de Huantajaya. Los indígenas de la región habían tenido la buena fortuna de no haber sido nunca incluidos en la mita de Potosí o Huancavelica y, hasta el siglo XVIII, la demanda de su mano de obra se había requerido bajo la forma de trabajos en el campo o de servicios personales1.

En un esfuerzo por contrarrestar el declive de la producción de plata, la Corona había ampliado el ámbito de la mita al abrir la posibilidad de concederla a los que poseían minas fuera de Potosí y Huancavelica. En 1756, a la luz de estas nuevas concesiones, el general José Bacilio de la Fuente escribió al virrey del Perú solicitando una mita para sus minas de Huantajaya2. Aunque poseía las minas más productivas de la región, un descenso en los ingresos, acompañado de altos costos en la mano de obra, le habían hecho imposible incrementar sus operaciones. Bacilio llevó a cabo un esfuerzo especial para demostrar cómo intentaba aplicar las nuevas regulaciones para cubrir la asignación de la mita. La Corona había insistido en que los mitayos fueran solamente asignados a lugares cuyo clima y medioambiente no fueran en detrimento de su bienestar, que sus pueblos de origen no estuvieran situados a más de 18 leguas de su trabajo. Bacilio proveyó testimonios jurados procedentes de los residentes más importantes de Tarapacá sobre la veracidad de estas condiciones. A pesar de ello, la Corona tardó más de dos años en concederle su petición de 50 mitayos (BB-HMTP: f. 1-71).

En Tarapacá, un Corregidor poco colaborador había retrasado la implementación de la mita durante tres años simplemente por rehusar responder a Bacilio. El propietario de la mina tuvo que esperar la llegada de un nuevo Corregidor para comenzar el proceso de implementación. En noviembre de 1761, el notario provincial fue autorizado a realizar un viaje a los pueblos para notificar a los kurakas que éstos necesitaban comenzar la conscripción. Aunque todos los kurakas o sus principales expresaron su acuerdo ante el notario, el kuraka de Sibaya, don José Lucaya, también escribió al Corregidor enumerando una serie de condiciones que debían darse antes de dar su consentimiento a la petición de la mita. Su principal objeción consistía en el hecho de que la mina estaba situada en

1 Para una completa descripción de las condiciones socioeconómicas durante el periodo colonial, véanse los trabajos de Villalobos (1979) y Trelles Arestegui (1991).

2 La principal fuente de este estudio es el documento de 105 páginas dentro del libro de registros de Bacilio, localizado en la Biblioteca Bancroft de la Universidad de California (Berkeley) bajo la rúbrica «Huantajaya Mine Tarapacá, Perú. Documents relating to Indian labor: 1757-1767» en la sección de documentos especiales de la biblioteca Bancroft de la Universidad de California, Berkley, desde ahora BB-HMTP. El documento fue donado a la biblioteca Bancroft en 1878 por Don G. R. Ghiselin, quien decía haberlo encontrado enterrado en la mina de plata «Los Gilgeros» en Huantajaya (Tarapacá). Parte del libro de registros ha sido ya transcrito y publicado en Villlalobos (1979). 
el desierto y, por tanto, no reunía las condiciones medioambientales adecuadas para sus mitayos, por ser muy diferentes de las de sus pueblos. Asimismo, el salario diario necesitaba ser incrementado y pagado en plata, y los mitayos debían ir acompañados de sus familias. Por otra parte, el periodo de descanso entre los turnos también debía incrementarse, o de lo contrario los mitayos no tendrían tiempo suficiente para regresar a sus hogares y preparar sus tierras con el fin de conseguir una buena cosecha. Finalmente, y para resolver una cuestión práctica, uno de los mitayos tendría que regresar a los pueblos con las mulas que transportaban a los otros mitayos a las minas, pues las mulas no tenían donde pastar ni abrevar en la zona (BB-HMTP: f. 78-80).

Bacilio, enfadado, respondió ese mismo día acusando a don José Lucaya de retrasar deliberadamente el proceso y de exagerar los inconvenientes y problemas del trabajo en la mina. Era un acto «despreciable» por parte del kuraka sugerir que el salario era demasiado bajo o incluso solicitar su pago en plata. Asimismo se sentía confuso por la insistencia del kuraka en que las familias de los mitayos debieran acompañarles, pues Bacilio creía que dicha práctica estaba a discreción de cada mitayo; y por lo tanto, correspondía a cada uno decidir sobre dicha cuestión. Las últimas dos peticiones sobre la extensión del periodo de descanso para poder preparar la tierra, así como el hecho de que un mitayo se quedase al cuidado de las mulas fueron objeto de una gran reprimenda por parte de Bacilio. Dichas demandas eran tan obtusas y tan «absolutamente impertinentes» que ni siquiera merecían una respuesta. Bacilio imploró al Corregidor que ignorara las alegaciones de Lucaya por considerarlas sin base alguna. Era bien sabido que las necesidades de los mitayos eran mínimas y que cualquier dificultad económica era debido a su «propensión a la holgazanería». Llegados a este punto, el Corregidor determinó que éste era un problema que no se resolvería fácilmente y aprovechó la oportunidad para excusarse, argumentando que debía atender negocios de estado en Tacna. Fue así como relegó en el notario el deber de intercambiar las cartas entre los dos adversarios (BB-HMTP: f. 81-84).

Sin embargo, don José Lucaya no había hecho sino empezar y fue así como en los dos días posteriores se reunió con los otros kurakas de Sibaya para, de manera colectiva, responder a la carta de Bacilio. Sabiendo perfectamente que no gozaban de una fuerte posición con respecto a Bacilio, apelaron al Corregidor a que considerase su caso teniendo en cuenta las desafortunadas condiciones en las que se encontraban. Los indígenas, al ser pobres e indefensos, no contaban con otro recurso sino el de apelar a Dios, a la caridad cristiana de la Corona y al Corregidor para que aliviaran su miseria. En su carta volvieron a enumerar las deplorables condiciones de trabajo en la mina, lo que con certeza aseguraba que en pocos años caerían gravemente enfermos o perecerían. Incluso con un salario de ocho reales al día les sería difícil a los mitayos pagar su tributo, alimentar y vestir a sus familias. Asimismo, el no permitir a los mitayos el suficiente tiempo como para regresar a casa para cuidar de sus cultivos, los condenaría a una vida de pobreza. Sus pueblos estaban a cuarenta leguas y no habría tiempo suficiente como para dedicarse al cuidado de los campos y hacer el viaje de regreso en el periodo de descanso que se les había concedido. Sugirieron que para completar dicha mita preferían ser reasignados a trabajar en la azoguería de Tilivilca, situada en las estribaciones andinas, en lugar de ser asignados a las minas. Dicho emplazamiento les facilitaría proveerse de sus necesidades, pues allí podrían cultivar alimentos, a diferencia del inhóspito medioambiente de la región de las minas. Así mismo, aceptarían un salario diario de cinco reales, con un suplemento de un real por cada trabajo especial llevado a cabo. Los kurakas argumentaron que su trabajo en Tilivilca proporcionaría la misma cantidad de impuestos para el tesoro de la Corona que si trabajasen en la mita de Huantajaya, y que, por tanto, a ojos de la Corona no debería haber ningún impedimento para que el Corregidor los reasignara. Aunque su petición de 
indulgencia se enmarcaba como un ruego desde los «pobres, conquistados y humildes vasallos de la corona», los kurakas estaban llevando a cabo una provocadora petición de unas concesiones extraordinarias. Dicha petición finalizaba expresando su deseo de haber resultado convincentes pues había muchas más razones por las que no debían ir a Huantajaya pero sus «poco sofisticados y rudos modos» les prevenían de articular dichas razones (BB-HMTP: f. 85-89).

Temiendo que don José Lucaya hubiera exagerado demasiado, don Francisco Lucaya, el kuraka de Tarapacá, se presentó ante el Corregidor, quien ya había regresado de su visita a Tacna. Aunque don Francisco le había dado su consentimiento verbal sobre la mita al notario, había rechazado firmar con su nombre el acuerdo arguyendo que no sabía cómo escribir. Explotando la ambigüedad de su posición, se presentó ante el Corregidor para indicar de forma clara su deseo de acatar e incluso aún más importante, su deseo de presentarse como una figura conciliadora para mitigar el tono beligerante de don José. Don Francisco recordó al Corregidor que considerase a los sedientos, mal pagados y mal pertrechados indígenas que habrían de trabajar en la mina de Bacilio, y que estaba situada a más distancia de sus pueblos que las dieciocho leguas estipuladas, como apuntó de manera casi espontánea. Aun siendo una violación de lo estipulado por la Corona, don Francisco no indicó nunca que tomaría ningún tipo de acción legal, aunque, claramente, la simple mención servía como amenaza encubierta, en caso de no aceptar los términos del kuraka.

Esta amenaza velada era el as en la manga del kuraka en sus negociaciones, pues la sola idea de recurrir a la autoridad de la Corona, situada por encima de la del Corregidor, hubiera podido no solamente poner en peligro la posición de éste, sino exponer el falso testimonio de Bacilio y sus testigos, pues todos habían afirmado en sus declaraciones juradas que los pueblos de los mitayos no se encontraban lejos de la mina y que allí gozarían de un clima similar, cosa que en ambos casos era falso. Es así que las relaciones personales y comerciales de Bacilio con dichos testigos, que a su vez eran distinguidos residentes de Tarapacá, hubieran podido verse seriamente afectadas de haber decidido los kurakas apelar a una autoridad superior. Desde una posición un poco más fuerte ahora, don Francisco continuó para calmar aquello que sin duda alguna comenzaba a resultar una gran alarma para el Corregidor. A fin de cuentas, ino sería en beneficio de todos el que la mita fuera reasignada a la azoguería que Bacilio poseía en Tilivilca? La azoguería se encontraba en un cañón no lejos de los pueblos y dentro de las dieciocho leguas estipuladas por la Corona. Don Francisco de esta manera intentaba evitar cualquier confrontación para proveer una clara y mejor solución para todos, pues Bacilio no tendría que aceptar las peticiones de un incremento de salario; los indígenas estarían más cerca de sus casas, pudiendo gozar así de unas mejores condiciones de trabajo y, finalmente, los kurakas no tendrían que verse obligados a la necesidad de iniciar un litigio por no estar de acuerdo con las peticiones de Bacilio (BB-HMTP: f. 90-91).

Dicha sugerencia, incluida la velada amenaza de acción legal, terminó por aplacar a Bacilio, pues su respuesta — un día después - reflejaba el deseo de zanjar la cuestión de una vez por todas. Finalmente, los razonamientos en frío comenzaban a prevalecer. Aunque en ocasiones exhibió algún tipo de sarcasmo en sus respuestas, éstas presentaban en general un sentimiento de resignación ante el hecho de que el curso de los acontecimientos comenzaba a sobrepasarle. Finalmente aceptó que la mita debía ser trasladada a su azoguería en Tilivilca. Después de todo, él también podía compadecerse de los indígenas. Fue así como, de manera un tanto ácida, Bacilio afirmó que si la mina de Huantajaya no podía ser trabajada por otra mita, entonces, «iDios no quiera!», se vería forzado a cerrarla. 
Previendo una rápida solución a la cuestión, el Corregidor decidió convocar una reunión para concluir el acuerdo y determinar un salario justo. Los kurakas decidieron aceptar la oferta de Bacilio de cuatro reales, un real menos del que habían solicitado anteriormente, al comprender que los mitayos en las azoguerías del Potosí recibían una compensación similar. Por su parte, Bacilio acordó permitir a los mitayos un tiempo extra para regresar a sus pueblos con el fin de cuidar de sus tierras. Asimismo, se les otorgaría tiempo para regresar a sus pueblos en pascuas para poder participar así en la elección de sus alcaldes. Por último, las condiciones de la mita se rebajaron a un periodo de dos meses y fue así como Bacilio finalmente consiguió su mita, aunque ésta no fuera sino la sombra de la que se le había concedido cinco años antes (BB-HMTP: f. 95-102).

La reasignación de la mita de Tilivilca se llevó a término sin mayores incidentes. Cinco años después, en diciembre de 1766, Bacilio se reunió con el nuevo Corregidor para renovar y revisar los términos de dicha mita una vez más. En la reunión general, los kurakas indicaron su satisfacción con la manera en la que la mita se estaba llevando a cabo y no pusieron ningún reparo para su extensión, a pesar de solicitar que su salario les fuera pagado enteramente en plata, petición a la que Bacilio no puso objeción alguna. El Corregidor pasó entonces a preguntar a cada uno de los kurakas de manera individual si tenían alguna otra cuestión que resolver con Bacilio, a lo que cada uno le respondió negativamente pues Bacilio era una persona de gran conciencia y caridad cristiana que velaba por ellos como si éstos fueran sus hijos (BB-HMTP: f. 103-105).

Después de cinco años de trabajo conjunto, Bacilio y los kurakas parecían haber desarrollado una relación más dialogante en la que Bacilio se mostraba ahora mucho más relajado, o quizás resignado, a las peticiones de reajustar los salarios. Por su parte, los kurakas accedieron sin más a la extensión de la mita. Aunque en este caso concreto los kurakas partían con ventaja, decidieron formular sus peticiones con exquisito cuidado pues eran muy conscientes de su relativa falta de poder en la sociedad colonial. Las concesiones que los kurakas fueron capaces de conseguir de Bacilio deben atribuirse a su tenacidad y su habilidad política. Asimismo, su estrecha relación con el Corregidor les reportó dividendos. Las noticias de Tarapacá indican cómo, desde su concepción, el repartimiento de mercancías nunca había tenido un gran éxito para su Corregidor, pues daba pérdidas en fechas tan tardías como 1778. La implementación del repartimiento de mercancías debería haber sido una fuente de ingresos para el Corregidor. Dicho repartimiento ensalzaba el poder del Corregidor a la hora de forzar la venta de una concesión, normalmente de menaje del hogar, a los indígenas de la provincia. El Corregidor compraba primero los utensilios a precio de mercado y se aprovechaba de su posición de autoridad para forzar a los indígenas a comprar dichos utensilios a precios muy inflados. Esto había causado un gran resentimiento en otras partes del Virreinato donde de manera habitual se producían revueltas contra el Corregidor (Gölte, 1980). Sin embargo, en Tarapacá, los kurakas pudieron proteger al Corregidor de cualquier descontento mediante la prevención de la excesiva explotación de los indígenas en el repartimiento de mercancías, tal y como atestiguan los documentos fiscales de la provincia. A cambio, los corregidores comprendieron que tendrían que prestar su apoyo tácito a los kurakas cuando éstos tuvieran que negociar con el Estado colonial. Los corregidores correspondieron con todo su apoyo en este caso. El primer Corregidor bloqueó de manera efectiva la implementación de la mita durante tres años y su sustituto jugó intencionadamente un papel pasivo durante el proceso de negociación. Junto a esto, el Virreinato pasaba por un momento delicado. La sucesión de rebeliones en la segunda mitad del siglo dieciocho había culminado con la rebelión de Túpac Amaru en 1781. Lo que se ha dado en llamar «la era de insurrecciones andinas», sin embargo, pasó por alto Tarapacá, tal y como lo demuestra el hecho de que no se informase nunca de ningún 
levantamiento. Es así como, en lugar de un momento de ruptura, Tarapacá representa un momento de continuidad en la historia del Virreinato. Las causas del malestar que habían dado lugar a la serie de rebeliones en otras partes estaban relacionadas con una debilitación del frágil equilibrio de fuerzas en la sociedad colonial, las que controlaban el trabajo indígena al servicio de la Corona y sus colonos. Dentro de esta dinámica, el kuraka jugaba un papel central y su desafío al viejo paradigma procedía de la implementación del repartimiento de mercancías y más peticiones de trabajo en un esfuerzo por encauzar la decadente producción de plata. En el caso de Tarapacá, sin embargo, fueron los kurakas hábiles en maniobras políticas quienes impidieron nuevas peticiones a los indígenas, bien se tratase del repartimiento de mercancías o de suplir la indeseable tarea de la mita en las minas. Era así como el pacto colonial» continuaba su supervivencia. Los kurakas de Tarapacá continuaron actuando como intermediarios para facilitar el acceso al trabajo indígena pero no podían pedir a sus mitayos ir más allá de lo que tradicionalmente se esperaba de ellos. Sus mitayos nunca habían trabajado en ninguna mina y tampoco sentían el deseo de hacerlo. Tan importante como esto era el hecho de que los mitayos no esperaban que sus kurakas les pidieran semejante trabajo. De esta forma, las prerrogativas tradicionales indígenas se mantenían lo cual resultaba en el continuado éxito en la relación de reciprocidad establecida entre kurakas e indígenas. En lugar del intolerable feudo de los kurakas de Tapacarí que habían forzado a los indígenas de Cochabamba a buscar mediación en las cortes coloniales para dirimir sus propias disputas, el caso de Tarapacá nos muestra a unos kurakas actuando en concierto con el fin de conseguir unas mejores condiciones para sus mitayos 3 . Asimismo, en lugar de encontrar a los indígenas apelando a las autoridades coloniales para que depusieran a sus kurakas, como sucedió en el caso de Chayanta, nociones de reciprocidad continuaron rigiendo la relación kuraka-indígena en Tarapacá (Serulnikov, 2003: 19-52). Un coeficiente extremadamente bajo entre indígenas y kurakas (32 a 1) en la provincia, uno de los más bajos del Virreinato, unido a la falta de una significante población forastera, contribuyeron por otra parte a mantener la estabilidad de dicha relación (Gölte, 1980: 155).

Si los kurakas de Tarapacá se habían sentido intimidados por el deterioro de las relaciones entre grupos en otras regiones, esto nunca se puso de manifiesto durante el proceso de negociación4. Su aparente pasividad y decepcionada estrategia de negociación encubría una posición agresiva que amenazaba con recurrir a la Corona si la cuestión no procedía según su parecer. La simulación de su ignorancia sobre las prácticas burocráticas generales de procedimiento o de su habilidad para firmar con sus nombres frustró el proceso de notificación e implementación dando tiempo a los kurakas para consultarse entre sí sobre la manera de llevar a cabo una estrategia común. En lugar de un uso indiscriminado o torpe de las «armas del débil» que caracterizan normalmente este tipo de acciones, su selectiva aplicación a manos de los kurakas en los momentos claves del proceso de negociación les hizo salir triunfantes en la eliminación de cualquier oposición a sus peticiones5. Un Corregidor

3 Desde comienzos de 1740, los caciques feudales de la villa de Tapacarí en Cochabamba envenenaron la vida privada durante aproximadamente veinte años, haciendo la situación tan intolerable que los indígenas a nivel particular solicitaron la mediación de los juzgados en sus propias disputas, antes que arriesgarse a que los caciques se involucraran (Larson, 1998: 152-159).

4 En 1754, ocho años antes de los acontecimientos aquí narrados, la rebelión indígena de la provincia de Arequipa, no lejos de Tarapacá, fue brutalmente reprimida. La rebelión, que había comenzado con el no pago del tributo personal de los indígenas, era una respuesta indígena al nuevo comportamiento de colaboración por parte de los kurakas con respecto de los nuevos corregidores borbones y que los indígenas percibían como una violación del tradicional comportamiento de los kurakas. Quizás la memoria de dicha rebelión mantuvo a los kurakas de Tarapacá más atentos a las peticiones de los indígenas (Salomon, 1987: 148-65).

5 Para un análisis sobre las diferentes formas de resistencia sin confrontación directa con la autoridad escogidas por los grupos oprimidos, véase Scott (1985). 
sumiso, cuya ruina fiscal se veía tan solo prevenida por el apoyo nominal del repartimiento de mercancías, hacía inefectivo el poder político en la provincia6. Por otra parte, un propietario minero financieramente inseguro, con miedo a un litigio y lo suficientemente desesperado como para reducir sus peticiones, neutralizaba cualquier desafío de tipo emprendedor. La inteligente explotación del kuraka de los puntos vulnerables del propietario de la mina y del Corregidor pudieron ser posibles solamente en un ambiente en el que al declive de la producción de plata se unía una escasez perenne de trabajo en unas zonas escasamente pobladas de una región desértica que daba así forma a sus realidades económicas. A pesar de todo, su habilidad en controlar el curso de los acontecimientos es digna de mención, pues aminoró el proceso de expansión de la mita fuera de Potosí y Huancavelica, lo que en sí no era poco.

La subversión de la autoridad colonial en esta «era de las insurrecciones andinas» no dio necesariamente lugar a una confrontación sangrienta. Los desafíos legales en las cortes coloniales para dirigir quejas, por ejemplo, fueron parte de esta «resistencia de adaptación» por la que los indígenas o los kurakas optaron en lugar de un conflicto directo. Lo que resulta interesante en el caso de Tarapacá es el hecho de que aunque nuevas peticiones de trabajo fueron resistidas con éxito, dicho éxito no se logró a través de una confrontación directa o recurso legal; más bien, los kurakas pudieron desafiar con éxito la imposición de la mina de la mita en gran parte gracias a que los participantes provinciales en este «pacto colonial» (el Corregidor, el propietario de la mina y el kuraka) continuaron reconociendo que dicho acuerdo daría sus dividendos solamente si todos y cada uno de ellos continuara jugando según las reglas, incluso en tiempos difíciles. Fue un acuerdo que continuó más allá de la extensión de la mita de Tilivilca en 1766. Ningún malestar patente o rebelión salió a la luz en Tarapacá en lo que quedó del siglo y fue así como la «era de las insurrecciones andinas» pasó desapercibida por esta región desértica.

\section{Referencias citadas}

\section{Fuentes primarias}

BB-HMTP - Libro de registros del General José Bacilio de la Fuente. Huantajaya Mine Tarapacá, Peru, Documents relating to Indian labor: 1757-1767; Berkeley: Biblioteca Bancroft de la Universidad de California.

\section{Fuentes secundarias}

GÖLTE, J., 1980 - Repartos y rebeliones. Túpac Amaru y las contradicciones de la economía colonial, 256 p.; Lima: Instituto de Estudios Peruanos.

6 El repartimiento de mercancías nunca había tenido mucho éxito para su corregidor. Bien a causa de oficiales honestos o por la imposibilidad de proveer con las mulas y las prendas que habían sido asignadas a la provincia, dicho repartimiento resultó ser uno de los menos lucrativos del Virreinato. Aún en 1778, la provincia daba pérdidas de casi cuatro mil pesos en esta cuenta (Moreno Cebrián, 1977: 127). 
LARSON, B., 1998 - Cochabamba 1550-1900. Colonialism and agrarian transformation in Bolivia, 422 p.; Durham/London: Duke University Press.

MORENO CEBRIÁN, A., 1977 - El corregidor de indios y la economía peruana del siglo XVIII, 801 p.; Madrid: Consejo Superior de Investigaciones Científicas, Instituto G. Fernández de Oviedo.

SALOMON, F., 1987 - Ancestor cults and resistance to the State in Arequipa (1748-1754). In: Resistance, rebellion, and consciousness in the Andean peasant world $18^{\text {th }}$ to $20^{\text {th }}$ centuries (Steve J. Stern, ed.): 148-165; Madison: The University of Wisconsin Press.

SERULNIKOV, S., 2003 - Subverting colonial authority. Challenges to spanish rule in eighteenth-century Southern Andes, 287 p.; Durham: Duke University Press.

SCOTT, J. C., 1985 - Weapons of the weak. Everyday forms of peasant resistance, 389 p.; New Haven: Yale University Press.

TRELLES ARESTEGUI, E., 1991 - Lucas Martínez Vegazo. Funcionamiento de una encomienda peruana inicial, 318 p.; Lima: Fondo Editorial de la Pontificia Universidad Católica del Perú.

VILLALOBOS R., S., 1979 - La economía de un desierto. Tarapacá durante la Colonia, 278 p.; Santiago de Chile: Ediciones Nueva Universidad. 